\title{
VEHICLE EMISSION COMPUTATION THROUGH MICROSCOPIC TRAFFIC SIMULATION CALIBRATED USING GENETIC ALGORITHM
}

\author{
Yun $\mathrm{Wei}^{1}$, Ying Yu ${ }^{2,4}$, Lifeng $\mathrm{Xu}^{3}$, Wei Huang ${ }^{4}$, Jianhua $\mathrm{Guo}^{2}$, Ying Wan ${ }^{6}$, Jinde Cao ${ }^{6}$ \\ ${ }^{1}$ Beijing Urban Construction Design and Development Group Co., Ltd, Beijing, China \\ ${ }^{2}$ Transportation Sensing and Cognition Research Center, Southeast University, Nanjing, China \\ ${ }^{3}$ Nanjing Vehicle Emission Regulatory Center, Nanjing, China \\ ${ }^{4}$ Intelligent Transportation System Research Center, Southeast University, Nanjing, China \\ ${ }^{5}$ School of Mathematics, Southeast University, Nanjing, China \\ E-mail:seuhwei@126.com
}

Submitted: 14th January 2018; Accepted: 4th March 2018

\begin{abstract}
Vehicle emission calculation is critical for evaluating motor vehicle related environmental protection policies. Currently, many studies calculate vehicle emissions from integrating the microscopic traffic simulation model and the vehicle emission model. However, conventionally vehicle emission models are presented as a stand-alone software, requiring a laborious processing of the simulated second-by-second vehicle activity data. This is inefficient, in particular, when multiple runs of vehicle emission calculations are needed. Therefore, an integrated vehicle emission computation system is proposed around a microscopic traffic simulation model. In doing so, the relational database technique is used to store the simulated traffic activity data, and these data are used in emission computation through a built-in emission computation module developed based on the IVE model. In order to ensure the validity of the simulated vehicle activity data, the simulation model is calibrated using the genetic algorithm. The proposed system was implemented for a central urban region of Nanjing city. Hourly vehicle emissions of three types of vehicles were computed using the proposed system for the afternoon peak period, and the results were compared with those computed directly from the IVE software with a trivial difference in the results from the proposed system and the IVE software, indicating the validity of the proposed system. In addition, it was found for the study region that passenger cars are critical for controlling $\mathrm{CO}$, buses are critical for controlling $\mathrm{CO}$ and VOC, and trucks are critical for controlling $\mathrm{NO}_{x}$ and $\mathrm{CO}_{2}$. Future work is to test the proposed system in more traffic management and control strategies, and more vehicle emission models are to be incorporated in the system.
\end{abstract}

Keywords: Environment protection, Microscopic traffic simulation, Genetic algorithm, IVE model, Emission evaluation

\section{Introduction}

Vehicle emissions are contributing to air quality degradation substantially, which is believed to be one of the most critical environmental issues facing the world [1]. Pollutant gases from vehicles appear as the single most important source of urban pollu- 
tant emissions in source apportionment studies [24], which is also an important cause of haze and photochemical smog pollution, seriously affecting the air quality of the city and human health [5-6]. In China, on-road vehicles emissions are preliminary calculated to be 4547.3 million tons, which contains $13.8 \% \mathrm{NO}_{x}$ emissions, $9.4 \% \mathrm{HC}$ emissions, $75.5 \% \mathrm{CO}$ emissions and $1.2 \% \mathrm{PM}$ emissions [7]. Therefore, many strategies are being implemented to reduce vehicle emissions, including new powertrains development, fuel replacement, technological improvements, strict traffic management regulation and emission standards [8]. To accurately assess the effectiveness and applicability of these measures in the urban area, emission inventories calculation is needed.

Over a long history, real-world measurement and model prediction method are two major measures used to compute emission inventories. However, real-world measurements, such as dynamometer testing, tunnel testing, remote sensing, and onboard instrumentation testing, have certain limitations such as limited test samples, fixed vehicle driving conditions, environmental impact instability [9]. By contrast, macroscopic and/or microscopic emissions model based on travel activity data both have been adopted in transportation environmental impact evaluation [10-11], showing many advantages such as low cost, repeatability, and sufficient sample size. For example, most emission inventories at the fleet level or at a regional level can be calculated using the COPERT model developed from the European MEET project [12-13]. In USA, IVE and MOVES are used more widely [1415], which are based on second-by-second speed data from traffic simulations or real-world measurements. Note that IVE model is developed for vehicle emissions computation in developing countries, covering more than 700 technology categories from small motorcycles to large trucks and buses [16]. In addition, each technology is assigned a default base emission factor, which can be modified by users according to local information. Clearly emission model is a good candidate for computing emission inventories.

Emissions calculation using the emission models needs vehicle driving characteristics (e.g., speed, acceleration and average speed) as the input data. Conventionally, these motor vehicle ac- tivity level data can be obtained using microscopic traffic simulation model. In these simulation models, an individual vehicle is modeled in detail for the entire trip, providing accurate traffic flow, travel time, and other traffic activity data. Typical traffic simulation software include CORSIM, SIGSIM, PARAMICS, VISSIM, TJTS, MicroSim, and NITS [17-22]. However, most of these microscopic simulation models lack relational database support to store simulation data so that the simulated traffic activity data cannot be applied effectively and directly for emission computation. In addition, the simulation should be calibrated adequately so that real-world situation can be captured in the simulation.

Therefore, the purpose of this paper is to develop an efficient integrated vehicle emission computation system through applying the technique of a relational database to a microscopic traffic simulation model. In doing so, second-by-second traffic activity data generated during the simulation can be organized and preprocessed into a prescribed input data for the emission computation module. In order to reflect the real-world situation, the simulation model is calibrated using Genetic Algorithm. Note that in this paper, the microscopic traffic simulation software is selected as Paramics and the emission model is selected as IVE model. In the rest of this paper, Section 2 presents an overview of related literature; Section 3 and Section 4 discuss the Paramics simulation software and IVE model, respectively; Section 5 presents the proposed system, which is implemented and tested in Section 6. In Section 7 , this paper concludes with summaries.

\section{Literature review}

In the field of emissions assessment, there is an emerging literature on integrating microscopic simulation models with emission models. Park et al. combined VISSIM with an existing velocitybased emission database (MODEM) and a Gaussian diffusion model to estimate the concentration of motor vehicle pollutants for various control measures [23]. Stathopoulos and Noland linked VISSIM with CMEM to evaluate the emissions for improving traffic efficiency [11]. Rakha and Ahn com- 
bined INTEGRATION with various models (e.g., car-following, lane changing, and vehicle dynamics) to estimate fuel consumption and emissions [24]. Servin et al. integrated PARAMICS with CMEM through the use of an Application Programming Interface (API) to predict emissions and fuel consumptions in real-time [25]. Xie et al. integrated MOVES and PARAMICS to evaluate the environmental impacts of three alternative transportation fuels [26]. Papson et al. utilized MOVES in conjunction with SYNCHRO to calculate emissions at congested and uncongested signalized intersections [27]. Some researchers created a software package (VIMIS) to integrate VISSIM and MOVES, which facilitates the converting of VISSIM files into MOVES files to evaluate various kinds of pollutants on the highway [28-29]. Zhao and Sadek used the simulated second-by-second vehicle trajectory data as the road driving behavior input required by MOVES emission model to analyze project level emissions [30]. Zhou et al. combined the Dynamic Traffic Assignment (DTA) model with MOVES to evaluate vehicle emissions and fuel consumption under different traffic control strategies [31].

From the above literature, though many studies have been conducted on integrating traffic simulation model and emission model, most of the simulation software does not support relational database based second-by-second simulated traffic activity data storage. In addition, current advanced emission models (e.g., IVE and MOVES) usually requires a large amount of input data. Therefore, it is difficult to apply the simulated traffic activity data directly into these vehicle emission models, resulting in less efficient vehicle emissions evaluation. In this end, we apply the database technique to store the vehicle activity data generated during Paramics simulation, and then organize and supply the data into an IVE calculation principle based emission computation module to compute vehicle emissions efficiently.

\section{Microscopic traffic simulation model-Paramics}

Paramics is a commercial microscopic traffic simulation software widely used in transportation planning, management, and decision-making [32]. The simulation toolset consists of eight modules: Modeller, Analyser, Processor, Designer, ODEstimator, Convertor, Monitor, and Programmer. Among them, Modeler is the core tool to construct a road network model, analyze simulation operation and generate second-by-second vehicle activity data. The Programmer provides the user with an application programming interfaces (API), which can be applied to implement traffic network control, simulation information acquisition, traffic control induction, and other extensions.

Paramics has an open architecture, providing about 700 interface functions which can be divided into four categories: override functions (QPO), extending functions (QPX), get functions (QPG), and set functions (QPS). QPO functions allow the users to replace the original core algorithm of Paramics software with customized code; QPX functions can be used to design the functional requirements of expansion modules; QPG functions are used to get real-time updates of Paramics simulation information; QPS functions allow the users to set values, states, and actions for objects in the Paramics model. Using Paramics, a large number of road network simulation data can be produced through constructing the road network model and running the simulation. In this way, vehicle activity data can be achieved using the simulation API Interface. However, Paramics does not supply direct storage of the simulation output in a relational database, which limits its support for applications like emission computation.

\subsection{The calculation principle of IVE model}

The IVE model is an on-road mobile source emissions model developed jointly by researchers at the International Sustainable System Research Center (ISSRC) and the University of California at Riverside (UCR)[14]. The IVE model modifies the influencing factors of the basic emission factors and takes into account the effects of various parameters, such as speed, acceleration, and road grade. The equations to calculate emissions are expressed in 
(1)-(3). The local factors used to correct the parameters in these equations are shown in Table 1.

$$
\begin{gathered}
Q_{[t]}=B_{[t]} \times K_{(1)[t]} \times K_{(2)[t]} \cdots \times K_{(n)[t]}, \\
Q_{\text {running }}=\bar{U}_{F T P} \times \frac{D}{\bar{U}_{C}} \times \sum_{t}\left\{f_{[t]} \times Q_{t} \times \sum_{d}\left[f_{d t} \times K_{[d t]}\right]\right\},
\end{gathered}
$$

$$
Q_{s t a r t}=\sum_{t}\left\{f_{[t]} \times Q_{t} \times \sum_{d}\left[f_{d t} \times K_{[d t]}\right]\right\}
$$

where $Q_{[t]}$ is the base emission for vehicle technology type $t ; B_{[t]}$ is the base emission rate for vehicle technology type $t ; f_{[t]}$ is the composition percentage for vehicle technology type $t ; f_{d t}$ is the driving or stopping composition percentage of vehicle technology type $t ; \bar{U}_{F T P}$ is the average speed in the LA 4 standard cycle condition, which is a constant; $D$ is vehicle mileage; $\bar{U}_{C}$ is the average speed in a particular driving condition; $K_{(x)[t]}$ is the adjustment parameter of different base emission rate; $K_{[d t]}$ is the driving condition or cold dip correction parameter; $Q_{\text {running }}$ is the driving emission; $Q_{\text {start }}$ is the starting emission.

A remarkable feature of the IVE model is to use vehicle activity characteristics, i.e., Vehicle Specific Power (VSP) and Engine Stress (ES), to describe the relationship between transient operating conditions and vehicle emissions. The equation is expressed as

$$
\begin{aligned}
V S P= & v[1.1 a+9.81(\arctan (\sin (\text { grade })))+0.132] \\
& +0.000302 v^{3},
\end{aligned}
$$

\section{EngineStress $=$ RPMIndex +0.08 PreaveragePower}

where $v$ is the real-time speed; $a$ is the realtime acceleration; grade is the grade of the road; PreaveragePower is the mean of VSP between the first 5 seconds and the first 25 seconds after starting the engine. RPMIndex is the speed index of engine that is the quotient of transient velocity and velocity division constant.
Considering the 20 intervals (bin) categorized by VSP ranges and three ranks (i.e., low, medium, and high) based on the value of ES, we define 60 VSP bins, each of which corresponds to an emission level in the IVE model. A detailed classification is defined in Table 2.

\subsection{Basic input parameters}

The parameters required for emission calculation are divided into four categories, including vehicle driving condition distribution, fleet technology distribution, basic emission factor, and fleet model adjustment coefficient.

Vehicle driving condition distribution refers to the distribution of VSP bins. Given the speed and acceleration of the motor vehicle, the distribution percentage of VSP bins can be calculated according to equations (4) and (5), and each VSP bin corresponds to a different adjustment factor.

Fleet technology distribution is defined as the proportion of various types of vehicles in the entire fleet. The IVE model has a total of 1372 types of established technologies and 45 types of nondefined technologies, which is categorized according to vehicle size, fuel type, vehicle usage, fuel delivery system, evaporation control system, and exhaust control system. A number (id) is assigned to the technical type of each vehicle.

Basic emission factor in IVE model is obtained specifically through comprehensive experiments in the United States under LA4 standard conditions. Therefore, for other regions, common urban vehicle types need to be matched with vehicle models in the IVE model before they can get the corresponding basic emission factors for each vehicle type. On this basis, the basic emission factor is modified using the adjustment factor.

Fleet model adjustment coefficient is defined as the adjustment factor of the specific vehicle type for the different types of air pollutants in a certain fleet, which is determined by combing local ambient conditions adjustment factor and the VSP bin adjustment factor. The parameters of local ambient conditions are shown in Table 3. 
Table 1. Local factors used for correcting the IVE parameters.

\begin{tabular}{lll}
\hline Local Factors & Fuel Quality Factors $K_{(F u e l)[t]}$ & Power and Driving Factors $K_{[d t]}$ \\
\hline Ambient temperature $K_{(T m p)[t]}$ & Overall quality of gasoline fuel & VSP \\
Ambient humidity $K_{(H m d)[t]}$ & Gasoline sulfur content & Road grade \\
Altitude $K_{(A l t)[t]}$ & Gasoline lead content & Air conditioning usage \\
I/M system $K_{(I M)[t]}$ & Gasoline benzene content & Start distribution \\
Regional baseline emission & Gasoline oxygen content & \\
adjustment $K_{(\text {Cntry })[t]}$ & Overall quality of diesel fuel & \\
& Diesel sulfur content & \\
\hline
\end{tabular}

Table 2. 60 VSP bins and correspondence between VSP and ES.

\begin{tabular}{lccc}
\hline $\mathrm{VSP}$ & Low $:-1.6<\mathrm{ES} \leq 3.1$ & Medium $: 3.1<\mathrm{ES} \leq 7.8$ & High : $7.8<\mathrm{ES} \leq 12.6$ \\
\hline$-80.0 \leq \mathrm{VSP}<-44.0$ & 0 & 20 & 40 \\
$-44.0 \leq \mathrm{VSP}<-39.9$ & 1 & 21 & 41 \\
$-39.9 \leq \mathrm{VSP}<-35.8$ & 2 & 22 & 42 \\
$-35.8 \leq \mathrm{VSP}<-31.7$ & 3 & 23 & 43 \\
$-31.7 \leq \mathrm{VSP}<-27.6$ & 4 & 24 & 44 \\
$-27.6 \leq \mathrm{VSP}<-23.4$ & 5 & 25 & 45 \\
$-23.4 \leq \mathrm{VSP}<-19.3$ & 6 & 26 & 46 \\
$-19.3 \leq \mathrm{VSP}<-15.2$ & 7 & 27 & 47 \\
$-15.2 \leq \mathrm{VSP}<-11.1$ & 8 & 28 & 48 \\
$-11.1 \leq \mathrm{VSP}<-7.0$ & 9 & 29 & 49 \\
$-7.0 \leq \mathrm{VSP}<-2.9$ & 10 & 30 & 50 \\
$-2.9 \leq \mathrm{VSP}<1.2$ & 11 & 31 & 51 \\
$1.2 \leq \mathrm{VSP}<5.3$ & 12 & 32 & 52 \\
$5.3 \leq \mathrm{VSP}<9.4$ & 13 & 33 & 53 \\
$9.4 \leq \mathrm{VSP}<13.6$ & 14 & 34 & 54 \\
$13.6 \leq \mathrm{VSP}<17.7$ & 15 & 35 & 55 \\
$17.7 \leq \mathrm{VSP}<21.8$ & 16 & 36 & 56 \\
$21.8 \leq \mathrm{VSP}<25.9$ & 17 & 37 & 57 \\
$25.9 \leq \mathrm{VSP}<30$ & 18 & 38 & 58 \\
$30 \leq \mathrm{VSP}<1000$ & 19 & 39 & 59 \\
\hline
\end{tabular}

Table 3. Local conditions.

\begin{tabular}{ll}
\hline Category & Detailed information \\
\hline Local environmental conditions & $\begin{array}{l}\text { Time, date, altitude, } \mathrm{I} / \mathrm{M} \text { system, } 27^{\circ} \mathrm{C} \text { air conditioning } \\
\text { use rate, road grade, humidity, temperature, etc. }\end{array}$ \\
$\begin{array}{l}\text { Fuel characteristics } \\
\text { Overall quality, sulfur, lead, benzene, oxygen content, etc. } \\
\text { Total running mileage or time, number of starts, driving mode } \\
\text { information, average speed, engine hot dip time, etc. }\end{array}$ \\
\hline
\end{tabular}




\subsection{IVE model application software}

Based on previous discussions, there has been an application software developed to support the application of IVE model. The emissions of various pollutants are calculated mainly through using three data input interfaces, including location interface, fleet interface, and base adjustment interface. The input data of location interface contains local environmental conditions, fuel characteristics, and driving behavior; fleet interface mainly contains the properties of vehicles, including vehicle type, fuel type, car weight, mileage, etc.; the data input to the base adjustment interface is the adjustment factor for base emission factors of each pollutant. Figure 1 shows an example of the location interface. Note that, repeated calculation of exhaust emissions is usually needed for evaluating various types of traffic control measures. Therefore, it is clear that the use of IVE software will be complex, time-consuming, and laborious.

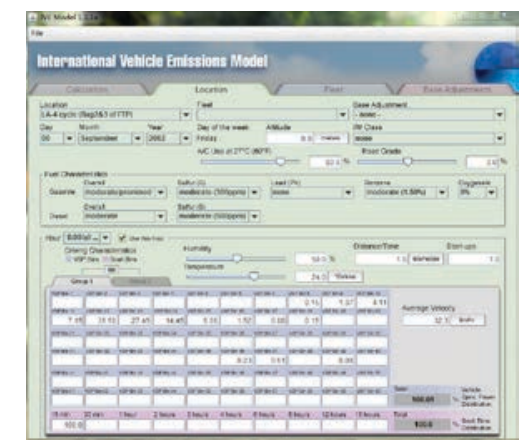

Figure 1. Location input interface example.

\section{Proposed Integrated Emission Computation System}

In this Section, two major parts, i.e., system framework design and calculation process, are described in detail for the proposed integrated emission computation system.

\subsection{System framework design}

The framework of the proposed system describes the components and the interaction of the components in the system, which is the key to integrating the microscopic traffic simulation model and the emission model. The layered design of the framework is adopted using the traditional traffic simulation framework combined with the current vehicle emissions assessment requirements, which is shown in Figure 2.

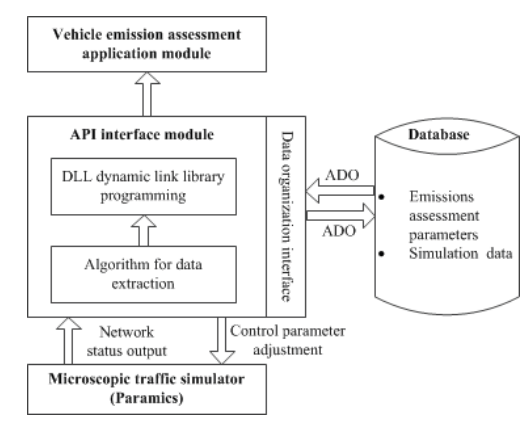

Figure 2. Proposed system framework.

Figure 2 shows the four main modules of the proposed system, namely the microscopic traffic simulator (Paramics), API interface module, database, and vehicle emissions assessment application module, as well as the relationship between different modules. Microscopic traffic simulator module can simulate the real-world traffic conditions and generate second-by-second vehicle activity data. These data can be extracted by the API interface module, which is the main control module of the proposed system. In this end, the Dynamic Linked Libraries (DLL) is applied to extract and store the vehicle activity data. At the same time, QPO function is called to adjust the control parameters of the microscopic traffic simulator. The database module uses ActiveX Data Objects (ADO) technology to realize the static and dynamic data interaction between the Paramics simulator and the relational database, i.e., the Microsoft Access database, and stores the static network information data and dynamic vehicle activity data generated in the simulation process. Therefore, the Access database contains two types of data tables, i.e., simulation data and emission assessment parameters, respectively. The detailed information of these data is shown in Table 4 and Table 5, respectively. Using the data stored in the database, the vehicle emissions assessment application module calculates vehicle emissions directly using the calculation principles discussed in Section 4.

\subsection{System operation process}

Based on the framework of the proposed system, the operation process of the proposed system is described in Figure 3. Clearly, this process includes four steps: vehicle type matching, simula- 
tion modeling and operation, evaluation algorithm of emission model, and fleet or regional emission calculation, which are described as below.

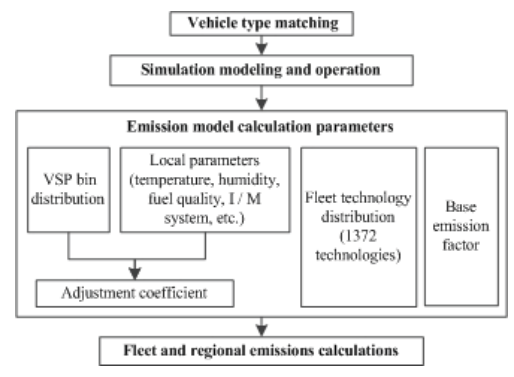

Figure 3. System operation process.

Vehicle type matching is the basis for regional motor vehicle emissions assessment. The main vehicle types of Paramics contain Car, Custom, LGV, OGV1 (8m), Coach, Bus, Minibus. The motor vehicle is divided into 1372 kinds of technology in the IVE model, and in reality, the actual calculation of emissions are mainly based on cars, buses, and trucks. Therefore, it is necessary to match the vehicle types (i.e., simulation vehicle type and the IVE vehicle type) to calculate the actual emissions, as shown in Table 6.

Simulation modeling and operation aims at generating vehicle activity data for the evaluation area. Before simulation, the basic data of the study region to be evaluated are collected, including the road attributes (i.e., geometric characteristics, road grade, lane composition, speed limit, etc.), timing parameters of the signals, and traffic flow data. Then, the road network modeling is completed using the Modeler tool provided by Paramics. Finally, the vehicle type and proportion data, road network OD data configuration and calibration are conducted. After developing and running the simulation model, the road network running data calculated by the Paramics simulator is obtained and stored by running the API interface program to provide the data source for motor vehicle emissions calculations.

Emission model evaluation parameters mainly contains activity data of motor vehicles and IVE model assessment parameters. In this step, the base emission factor and fleet technology distribution are stored in the relational database. In addition, VSP bin distribution data generated using the simulated second-by-second vehicle activity data and the local parameters stored in the relational database are used to compute the adjustment coefficients. In this way, the input data set can be generated for computing vehicle emissions.

After obtaining all the necessary parameters, fleet or regional emission calculation can be conducted by applying the calculation principle of the IVE model presented in Section 4. Note that the fleet pollutant emission is calculated first, and the regional emissions are then computed through summing up all the fleet emissions.

\section{Empirical test and analysis}

In this Section, the proposed integrated vehicle emission computation system is validated through a field implementation in a selected study area, and the vehicle emissions are analyzed with respect to each typical pollutant.

\subsection{Study area, simulation model develop- ment, and data collection}

The core area of Xinjiekou in Nanjing is selected as the study area, which is surrounded by the arterial roads and secondary roads (i.e., Shanghai Road, Mochuo Road, Taiping North Road, Changbai Street, Jianye Road, Baixia Road, Guangzhou Road and Zhujiang Road). This area is about 5.06 $\mathrm{km}^{2}$ and the road network of the study area is shown in Figure 4. According to the selected region, the simulation model of the road network is developed in Paramics, containing 200 sections, 75 nodes, and 16 traffic zones.

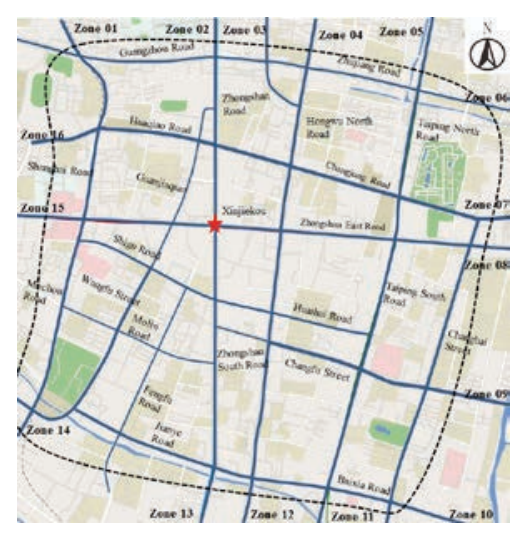

Figure 4. Selected study area in Nanjing City.

Traffic flow data are collected using video recording technique, with vehicle license plate data reduced for 11 intersections of the study area during morning and afternoon peak periods. The ve- 
Table 4. Summary of Paramics simulation data.

\begin{tabular}{ll}
\hline Paramics simulation parameters & Description \\
\hline Road network table & Basic configuration information of road network \\
Road sections table & Traffic and geometric characteristics of each section \\
Road segments table & Section number and the number of lanes \\
Road lanes table & Lane number and geometric characteristics of the lane \\
Signal lights table & Signal position and timing parameter configuration \\
Intersection nodes table & Intersection number and configuration \\
Traffic zone nodes table & Traffic zone characteristics and the total number of vehicles \\
OD pairs table & Origin and destination information of each vehicle \\
Route table & Route information between vehicle origin and destination \\
Detectors table & Location of the detectors \\
Traffic signs table & Traffic sign number and location information \\
Bus stations table & Location and information about the bus station \\
Vehicles table & Second-by-second vehicle location and activity data \\
\hline
\end{tabular}

Table 5. Summary of vehicle emissions assessment parameter data.

\begin{tabular}{|c|c|}
\hline Emissions assessment parameter & Description \\
\hline Car BER table & $\begin{array}{l}\text { Car types on the primary and secondary roads } \\
\text { and base emission rate of pollutants }\end{array}$ \\
\hline Bus BER table & Bus types and base emission rate of pollutants \\
\hline Truck BER table & Car types and base emission rate of pollutants \\
\hline Car technical distribution table & $\begin{array}{l}\text { Major/secondary road car index } \\
\text { and technology distribution ratio }\end{array}$ \\
\hline Bus technical distribution table & Bus index and technology distribution ratio \\
\hline Truck technical distribution table & Truck index and technology distribution ratio \\
\hline Car adjustment coefficient table & $\begin{array}{l}\text { Pollutant adjustment factor for car emissions } \\
\text { on the major/secondary road }\end{array}$ \\
\hline Bus adjustment coefficient table & Pollutant adjustment factor for bus emissions \\
\hline Truck adjustment coefficient & Pollutant adjustment factor for truck emissions \\
\hline
\end{tabular}

Table 6. Vehicle type matching classification.

\begin{tabular}{lll}
\hline Common vehicle type & Paramics vehicle type & IVE vehicle technology type number $($ id $)$ \\
\hline Car & Type22, Car & $117,118,119$ \\
& Type23, Car & $173,181,189$ \\
Bus & Type21, Coach & $1085,1087,1088$ \\
& Type31, Coach & 1133,1141 \\
Truck & Type17-20, LGV & $1085,1087,1088$ \\
& Type26-28, LGV & $1080,1081,1082$ \\
& Type25, OGV1 & 1083,1084 \\
\hline
\end{tabular}


hicle license plate data are then processed to obtain the information on 15 vehicle types. Afterward, the vehicle type information collected from the study region are matched with those of Paramics, with the proportions of vehicle types shown in Table 7. The first and second columns of Table 7 represent the main vehicle types in Nanjing, with H1, H2, H3 for trucks, K1-K4 for buses, Q1 and Q2 for tractors, and Z1, Z2, Z3, Z5, Z7 for special work cars. The third column represents the vehicle types in Paramics, with LGV standing for light trucks, OGV1 standing for $8 \mathrm{~m}$ long medium trucks, and coaches standing for long distance buses.

\subsection{Simulation model calibration}

In order to reflect the real-world condition of the selected road network, the developed simulation model should be calibrated in that the traffic flow data generated by the simulation system will be close to the collected traffic flow data in real-world as much as possible. For this purpose, we adopt the genetic algorithm (GA) to determine the OD matrix as the input to the simulation system, which is implemented using Matlab. Note that GA is inherently an iterative procedure, and in each iteration, the fitness value of each individual will be computed based on the simulated result, which means that the Paramics simulator will be called to compute the fitness value for each individual through the entire calibration process. For this purpose, API should be used to conduct data interaction between Matlab and Paramics. The procedure of GA based simulation calibration is shown in Figure 5.

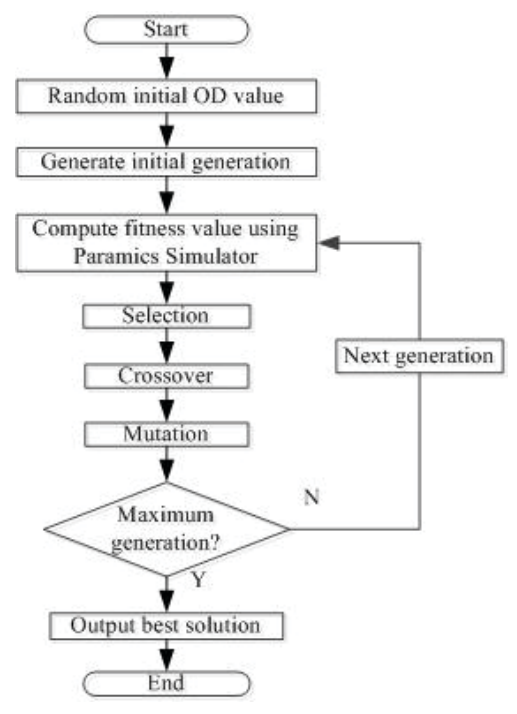

Figure 5. GA based simulation calibration procedure.

For the GA presented in Figure 5, the real number chromosome coding is adopted for representing the OD (Origin-Destination) matrix to be calibrated. In addition, for the GA operators, selection refers to selecting individuals from the previous generation with a certain probability to the new generation, crossover refers to exchanging two chromosomes selected to produce a new individual, and mutation refers to changing the chromosome of a selected individual to produce a new one. Furthermore, the difference between simulated traffic flow data and observed traffic flow data is used to define the fitness function, as in Equation (6).

$$
\text { Fitness }=\frac{10}{\max \left|o b s_{i}-\operatorname{sim}_{i}\right|+1},
$$

where $o b s_{i}$ is the observed traffic flow value of the section $i$ and $\operatorname{sim}_{i}$ is the simulated traffic flow value of section $i$.

Finally, the OD matrix with the largest fitness value in the output population is taken as the best solution. The final calibrated OD data is shown in Table 8 for the selected study region. Note that in this study, the population size is 40 , the crossover probability is 0.6 , the mutation probability is 0.01 , and the maximum number of generations is 50 .

\subsection{System validation}

For system validation, emission calculation parameters are firstly obtained, including a driving condition distribution, vehicle technology distribution, and other parameters. The driving condition and the distribution data of VSP bins are calculated using the simulated speed and acceleration of each vehicle (i.e., car, bus, and truck). The distribution of vehicle technologies is obtained by investigating the distribution of cars, buses, and trucks in the study area and matching these vehicle types with those of the IVE model. Other parameters include the vehicle type adjustment factor and the basic emission factor obtained using the survey data of the basic information about Nanjing City (shown in Table 9).

After collecting all the parameters, carbon monoxide (CO), volatile organic compounds (VOC), nitrogen oxides $\left(\mathrm{NO}_{x}\right)$, and carbon dioxide $\left(\mathrm{CO}_{2}\right)$ emissions for the afternoon peak hour of the 
Table 7. Vehicle type matching classification.

\begin{tabular}{lllll}
\hline Vechile & Vehicle type & Paramics vehicle type & Quantity & Ratio(\%) \\
\hline Truck & H1 & Type17-18, LGV & 687 & 5.76 \\
& H2 & Type19, LGV & 235 & 1.97 \\
\multirow{5}{*}{ Bus } & H3 & Type20, LGV & 121 & 1.01 \\
& K1 & Type21, Coach & 1179 & 9.88 \\
& K2 & Type31, Coach & 119 & 1.00 \\
& K3 & Type22, Car & 9179 & 76.95 \\
Tractor & K4 & Type23, Car & 64 & 0.54 \\
& Q1 & Type24, OGV2 & 170 & 1.43 \\
Special work car & Q2 & Type25, OGV1 & 3 & 0.03 \\
& Z1 & Type26, LGV & 1 & 0.01 \\
& Z2 & Type27, LGV & 48 & 0.40 \\
& Z3 & Type28, LGV & 14 & 0.12 \\
& Z5 & Type29, LGV & 99 & 0.83 \\
Total & Z7 & Type30, LGV & 10 & 0.08 \\
\hline
\end{tabular}

Table 8. Illustration of the calibrated OD matrix for study region.

\begin{tabular}{ccccccccccc}
\hline Zone & 1 & 2 & 3 & 4 & $\ldots$ & 13 & 14 & 15 & 16 & Sum \\
\hline 1 & & 0 & 265 & 451 & $\ldots$ & 4 & 421 & 81 & 64 & 2630 \\
2 & 0 & & 0 & 270 & $\ldots$ & 364 & 429 & 358 & 116 & 3628 \\
3 & 265 & 0 & & 0 & $\ldots$ & 177 & 444 & 302 & 12 & 3224 \\
4 & 451 & 270 & 0 & & $\ldots$ & 390 & 244 & 217 & 304 & 4446 \\
5 & 216 & 271 & 356 & 0 & $\ldots$ & 218 & 110 & 442 & 55 & 3040 \\
6 & 8 & 400 & 152 & 239 & $\ldots$ & 218 & 113 & 73 & 204 & 2287 \\
7 & 128 & 185 & 331 & 85 & $\ldots$ & 25 & 268 & 89 & 442 & 2600 \\
8 & 99 & 98 & 163 & 440 & $\ldots$ & 25 & 381 & 317 & 274 & 2826 \\
9 & 96 & 484 & 204 & 422 & $\ldots$ & 46 & 174 & 312 & 185 & 3089 \\
10 & 392 & 232 & 407 & 449 & $\ldots$ & 297 & 231 & 164 & 104 & 3483 \\
11 & 351 & 189 & 367 & 477 & $\ldots$ & 121 & 320 & 401 & 220 & 3301 \\
12 & 54 & 232 & 44 & 458 & $\ldots$ & 0 & 459 & 500 & 478 & 2997 \\
13 & 4 & 364 & 177 & 390 & $\ldots$ & & 0 & 490 & 62 & 2437 \\
14 & 421 & 429 & 444 & 244 & $\ldots$ & 0 & & 0 & 235 & 3829 \\
15 & 81 & 358 & 302 & 217 & $\ldots$ & 490 & 0 & & 0 & 3746 \\
16 & 64 & 116 & 12 & 304 & $\ldots$ & 62 & 235 & 0 & & 2755 \\
Sum & 2630 & 3620 & 3224 & 4446 & $\ldots$ & 2437 & 3829 & 3746 & 2755 & 50318 \\
\hline
\end{tabular}

Table 9. Basic information of Nanjing City.

\begin{tabular}{lllll}
\hline Index & Value & Fuel & Index & Range \\
\hline Ambient temperature & $20^{\circ} \mathrm{C}$ & Gasoline quality & Overall quality & Medium/premixed \\
Relative humidity & $80 \%$ & & Sulfur content & $300 \mathrm{ppm}$ \\
Average altitude & $22 \mathrm{~m}$ & & Lead content & None $(<0.013)$ \\
$\begin{array}{l}\text { Average slope of road } \\
27^{\circ} \mathrm{C} \text { air conditioning }\end{array}$ & $0 \%$ & & Benzene content & Medium $(1.5 \%)$ \\
opening ratio & & & Oxidation rate & $0 \%$ \\
I/M & Idle speed monitoring & Diesel quality & Overall quality & Medium \\
& & & Sulfur content & $500 \mathrm{ppm}$ \\
\hline
\end{tabular}


study area are calculated using the proposed system, with the results shown in Table 10. Using the same set of parameters, direct application of the IVE software, i.e., input the obtained parameters using the IVE interfaces manually is also conducted, with the results shown in Table 11.

Through a comparison of Table 10 and Table 11 , it can be found that the emissions for all the pollutants and for all the vehicle types are almost identical, with a difference around $1 \mathrm{~kg}$, indicating that the proposed system can calculate favorably vehicle emissions. Therefore, considering the automatic computation ability of the proposed system, the efficiency of computing vehicle emissions is highly improved, supporting repeated runs of vehicle emission calculation, which is useful when a large amount of vehicle emission calculation are inevitable for comparing different vehicular traffic management and control measures.

\subsection{Emission analysis}

Using the proposed vehicle emission computation system, emissions are analyzed for the selected study region. First, on reading Table 10, it can be seen that the $\mathrm{CO}$ and $\mathrm{VOC}$ emissions of buses are much higher than those of cars and trucks. In addition, buses and trucks emit a much higher level of $\mathrm{NO}_{x}$ than cars. Moreover, the total emissions are significantly different, with the highest as $\mathrm{CO}_{2}$, followed by $\mathrm{NO}_{x}, \mathrm{CO}$, and VOC. When looking into the sharing rate of each vehicle type as shown in Figure 6, it can be seen that for $\mathrm{CO}$ and VOC, the contribution rate of buses reached $46 \%$ and $58 \%$, respectively, indicating that buses are the focus of $\mathrm{CO}$ and VOC emission control; for $\mathrm{NO}_{x}$ and $\mathrm{CO}_{2}$, trucks have the highest contribution rate as $66 \%$ and $61 \%$, respectively; for CO, cars have a third of the total CO emissions. Given the above information, clearly, passenger cars are critical for controlling $\mathrm{CO}$, buses are critical for controlling $\mathrm{CO}$ and VOC, and trucks are critical for controlling $\mathrm{NO}_{x}$ and $\mathrm{CO}_{2}$, for this urban area.

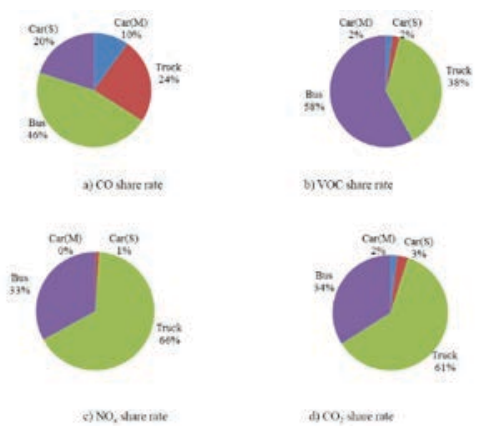

Figure 6. Pollutant emission sharing rates for the study area.

\section{Conclusion}

Air pollution caused by motor vehicles has been a growing concern for many counties. For developing vehicular traffic management and control measures, repeated calculation of vehicle emission would be inevitable. Currently, combining microscopic traffic simulation model with the vehicle emission model is a promising approach. However, due to the lack of relational database support for many traffic simulation models and the complex structure of currently used advanced vehicle emission models, the computation of vehicle emissions is still laborious and time-consuming. In addition, for reflecting the real-world situation, the simulation model should be calibrated adequately.

Targeting these issues, this work proposed an integrated vehicle emission computation system that provides an effective and efficient way of computing vehicle emissions. In this system, the relational database is built into the simulation environment that is calibrated using the genetic algorithm, and the vehicle emission model is implemented as a built-in emission computation module. In addition, the parameters needed for emission calculation will be prepared based on information stored in the relational database. Therefore, automatic vehicle emission computation can be conducted on the simulated road network model. In this way, traffic control and management measures, such as signal timing optimization can be evaluated easily in terms of vehicle emissions in various settings. Note that in this study, the microscopic traffic simulator is selected as Paramics, and the vehicle emission model is selected as the IVE model. In addition, a genetic algorithm is used to calibrate the simulation model so that the simulated vehicle activity data could be 
Table 10. Vehicle emissions calculated using proposed system (Unit: kg).

\begin{tabular}{lllll}
\hline Vehicle type & $\mathrm{CO}$ & $\mathrm{VOC}$ & $\mathrm{NO}_{x}$ & $\mathrm{CO}_{2}$ \\
\hline Car(major road) & 1284.3 & 42.5 & 90.0 & 54785.3 \\
Car(secondary road) & 2426.39 & 55.46 & 148.08 & 69435.83 \\
Bus & 5668.0 & 1265.0 & 13995.0 & 817216.0 \\
Truck & 2993.0 & 834.7 & 27881.7 & 1481215.6 \\
Sum & 12371.7 & 2197.7 & 42114.8 & 2422652.7 \\
\hline
\end{tabular}

Table 11. Vehicle emissions calculated using IVE software (Unit: $\mathrm{kg}$ ).

\begin{tabular}{lllll}
\hline Vehicle type & $\mathrm{CO}$ & $\mathrm{VOC}$ & $\mathrm{NO}_{x}$ & $\mathrm{CO}_{2}$ \\
\hline Car(major road) & 1284.6 & 42.79 & 90.28 & 54785.81 \\
Car(secondary road) & 2426.39 & 55.46 & 148.08 & 69435.83 \\
Bus & 5670.62 & 1267.18 & 13996.0 & 817216.0 \\
Truck & 2993.0 & 834.7 & 27882.27 & 1481216 \\
Sum & 12375.1 & 2200.5 & 42116.6 & 2422657.3 \\
\hline
\end{tabular}

close to the real-world vehicle activity. This is critical to ensure the accurate computation of the motor vehicle emissions.

In order to prove the practicality of the proposed system, this study uses the core area of Nanjing City as an example to establish and calibrate the road network model so as to calculate emission inventory of vehicles for the afternoon peak hour. Through comparing the computed results from the proposed system and the IVE software, it is found that the proposed system can generate comparable vehicle emission results as those of the IVE software, indicating the validity of the proposed system. In addition, on looking at the emission results, it is found for the selected urban region that buses are the focus for controlling $\mathrm{CO}$ and $\mathrm{VOC}$, trucks are the focus of controlling $\mathrm{NO}_{x}$ and $\mathrm{CO}_{2}$, and cars are critical for controlling $\mathrm{CO}$.

With the increase in a number of motor vehicles in urban areas, environmental degradation and hence emission calculation and regulation will receive sustained attention. In this end, the proposed system is expected to be applied in evaluating various vehicular regulation measures. In addition, additional emission models, such as MOVES model, can be integrated into the system for further study.

\section{Acknowledgements}

This work is supported by the National Natural Science Foundation of China (Grant No.61573106).

\section{References}

[1] A. Kendall, L. Price, Incorporating time-corrected life cycle greenhouse gas emissions in vehicle regulations, Environmental Science and Technology, 46(5), 2012, 2557-2563.

[2] V. Franco, M. Kousoulidou, M. Muntean, L. Ntziachristos, S. Hausberger, P. Dilara, Road vehicle emission factors development: A review, Atmospheric Environment, 70(70), 2013, 84-97.

[3] N. Maykut, J. Lewtas, E. Kim, T. Larson, Source apportionment of PM2.5 at an urban improve site in Seattle, Washington, Environmental Science and Technology, 37(22), 2003, 5135-5142.

[4] X. Querol, M. Viana, A. Alastuey, F. Amato, T. Moreno, S. Castillo, P. Salvador, Source origin of trace elements in PM from regional background, urban and industrial sites of Spain, Atmospheric Environment, 41(34), 2007, 7219-7231.

[5] N. Janssen, G. Hoek, M. Simic-Lawson, P. Fischer, L. Bree, H. Brink, M. Keuken, R. Atkinson, H. Anderson, B. Brunekreef, F. Cassee, Black carbon as an additional indicator of the adverse health effects of airborne particles compared with PM10 and PM2.5, Environmental Health Perspectives, 119(12), 2011,1691-1699.

[6] R. Laumbach, H. Kipen, Respiratory health effects of air pollution: Update on biomass smoke and traffic pollution, Journal of Allergy and Clinical Immunology, 129(1), 2012, 12-3.

[7] People's Republic of China(PRC) Environmental Protection Agency, China motor vehicle pollution prevention and control annual report, Beijing, 2015. 
[8] European Commission, White paper - roadmap to a single European transport area-towards a competitive and resource efficient transport system - impact assessment, European Commission, Brussels, Belgium, 2011.

[9] T. Chang, S. Modzelewski, J. Norbeck, W. Pierson, Tunnel air quality and vehicle emissions, Atmospheric Environment, 15(6), 1981, 1011-1016.

[10] K. Ahn, H. Rakha, A. Trani, M. Aerde, Estimating vehicle fuel consumption and emissions based on instantaneous speed and acceleration levels, Journal of Transportation Engineering, 128(2), 2002, 182-190.

[11] F. Stathopoulos, R. Noland, Induced travel and emissions from traffic flow improvement projects, Transportation Research Record, No.1842, 2003, 57-63.

[12] L. Ntziachristos, Z. Samaras, Copert III, computer programme to calculate emissions from road transport, European Environment Agency, Copenhagen, 2000.

[13] J. Hickman, D. Hassel, R. Joumard, Z. Samaras, S. Sorenson, Methodology for calculating transport emissions and energy consumption, European Commission, Brussels, Belgium, 1999.

[14] ISSRC, IVE model users manual version 2.0 retrieved, International Sustainable Systems Research Center, La Habra, CA, 2014.

[15] United States Environmental Protection Agency(USEPA), MOVES 2004 users guidełdraft, Office of Transportation and Air Quality, US Environmental Protection Agency, Washington, DC, 2004.

[16] N. Davis, J. Lents, M. Osses, N. Nikkila, M. Barth, Part 3: Developing countries: Development and application of an international vehicle emissions model, Transportation Research Record, Np.1939, 2005, 155-165.

[17] A. Halati, H. Lieu, S. Walker, CORSIM-corridor traffic simulation model, The Annual Meeting of the Transportation Research Board, Washington DC, 1997.

[18] M. Madireddy, B. Coensel, A. Can, B. Degraeuwe, B. Beusen, I. Vlieger, D. Botteldooren, Assessment of the impact of speed limit reduction and traffic signal coordination on vehicle emissions using an integrated approach, Transportation Research Part D, 16(7), 2011, 504-508.

[19] PTV, VISSIM user manual-version 3.70, Karlsruhe, Germany, 2003.

[20] Z. Zhou, Application of TJTS simulation system, China Journal of Highway and Transport, 14(z1), 2001, 92-96.
[21] J. Duan, Microscopic traffic simulation of high speed road, Journal of Highway and Transportaion Research and Development, 15(3), 1998, 21-24.

[22] Z. He, Z. Yang, X. Jiang, J. Miao, W. Chen, Research on general simulation system of urban traffic, China Journal of Highway and Transport, 16(1), 2003, 95-98.

[23] J. Park, R. Noland, J. Polak, Microscopic model of air pollutant concentrations: Comparison of simulated results with measured and macroscopic estimates, Transportation Research Record, No.1750, 2001, 64-73.

[24] H. Rakha, K. Ahn, Integration modeling framework for estimating mobile source emissions, Journal of Transportation Engineering, 130(2), 2004, 183-193.

[25] O. Servin, K. Boriboonsomsin, M. Barth, An energy and emissions impact evaluation of intelligent speed adaptation, IEEE conferenc on Intelligent Transportation Systems, Toronto, Canada, 2006, 1257-1262.

[26] Y. Xie, M. Chowdhury, P. Bhavsar, Y. Zhou, Integrated tool for modeling impact of alternativefueled vehicles on traffic emissions: Case study of Greenville, South Carolina, The Annual Meeting of the Transportation Research Board, Washington DC, 2011.

[27] A. Papson, S. Hartley, K. Kuo, Analysis of emissions at congested and uncongested intersections with motor vehicle emission simulation 2010, Transportation Research Record, No.2270, 2012, 124-131.

[28] H. Abou-Senna, E. Radwan, K. Westerlund, C. Cooper, Using a traffic simulation model (VISSIM) with an emissions model (MOVES) to predict emissions from vehicles on a limited-access highway, Journal of the Air and Waste Management Association, 63(7), 2013, 819-831.

[29] H. Abou-Senna, E. Radwan, Vissim/moves integration to investigate the effect of major key parameters on $\mathrm{CO}_{2}$ emissions, Transportation Research Part D, 21(2), 2013, 39-46.

[30] Y. Zhao, A. Sadek, Computationally-efficient approaches to integrating the moves emissions model with traffic simulators, Procedia Computer Science, 19, 2013, 882-887.

[31] X. Zhou, S. Tanvir, H. Lei, J. Taylor, B. Liu, N. Rouphail, H. Frey, Integrating a simplified emission estimation model and mesoscopic dynamic traffic simulator to efficiently evaluate emission impacts of traffic management strategies, Transportation Research Part D, 37(12), 2015, 123-136.

[32] Quadstone, Quadstone PARAMICS V4.2 processor user manual, Edinburgh, Scotland, 2003 


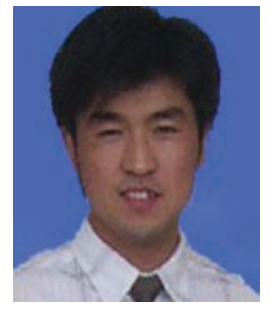

Yun Wei receives B.S. from Nanjing University of Aeronautics and Astronautics in 2005, MS from Southeast University in 2008, and Ph.D. from Southeast University in 2013. He is the vice director of the Urban Railway Green and Safe Construction National Engineering Laboratory. His research field includes intelligent vision analysis and pattern recognition. He received the Beijing Science and Technology New Star Award and the Exceptional Talent Award of Beijing. He is a member of the Comprehensive Intelligent Transportation Systems Technical Committee of Chinese Association of Automation.

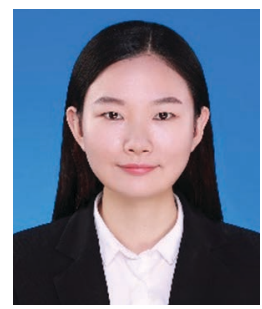

Ying Yu received her bachelor's degree in traffic engineering from Anhui Jianzhu University, Anhui, P.R. China. She is currently pursuing the master's degree at the Intelligent Transportation System Research Center, Southeast University, Nanjing, China. She is currently involved in the research of vehicle emissions evaluation method, especially on combination of microscopic traffic simulation and vehicle emissions calculation model.

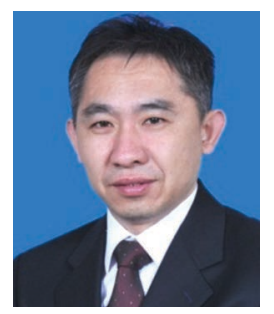

Lifeng $\mathbf{X u}$ is the Deputy Chief Engineer of the Nanjing Vehicle Emission Regulatory Center and the external master student advisor for Southeast University. Since 2008, he works primarily on motor vehicle emission mitigation regulations, conducting state, provincial, or municipal funded studies with institutions such as American Energy Foundation, Nanjing University, or Southeast University. Previously, he worked on integrated monitoring and analysis of water, atmosphere, and noise.

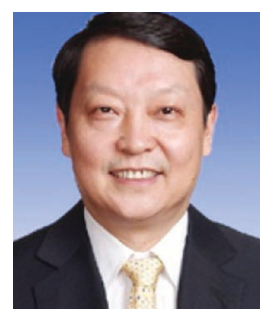

Wei Huang is a distinguished professor at Southeast University, Nanjing, Jiangsu, P.R. China. He is a member of Chinese Academy of Engineering. He enjoys the State Council special allowance and receives supports from the New Century Talent Program, the National Outstanding Mid-aged Experts
Program, the National Talents Engineering Program, and the Yangtze Scholar Program. He is one of the forerunners in the research fields of long-span steel bridge pavement and intelligent transportation systems of China. As the leading awardee, he receives 26 awards from both the national and provincial level. He published 13 books.

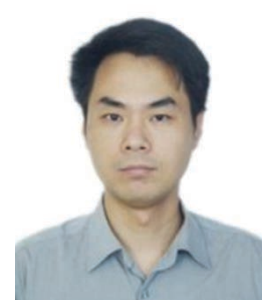

Jianhua Guo is a professor in Southeast University, Nanjing, Jiangsu, P.R. China. His major research fields include intelligent transportation system applications, traffic simulation and control, statistical time series analysis, and discrete choice modeling. He received research funds from the Chinese Natural Science Foundation, Ministry of Science and Technology of China, and provincial agencies. He serves as reviewer for Chinese National Science Foundation and international journals.

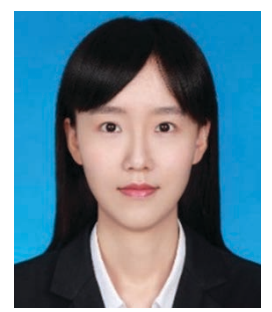

Ying Wan received the B.S. degree from Anhui Normal University, Wuhu, China, in 2012. She is currently working toward the Ph.D. degree at Southeast University, Nanjing, P.R. China. Her current research interests include neural networks, cooperative control of multi-agent systems and cyberphysical systems.

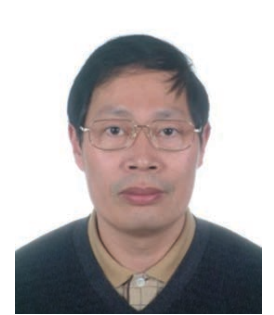

Jinde Cao is a Distinguished Professor, the Dean of School of Mathematics and the Director of the Research Center for Complex Systems and Network Sciences at Southeast University. He is Associate Editor of the Journal of the Franklin Institute, Neurocomputing, the IEEE Transactions on Cybernetics, Differential Equations and Dynamical Systems, Mathematics, and Computers in Simulation, and Neural Networks. He is a Fellow of IEEE, a Member of the Academy of Europe, a Fellow of Pakistan Academy of Sciences, and a member of European Academy of Sciences and Arts. He is Highly-Cited Researcher in three fields named by Thomson Reuters. 